\title{
WHO guidelines for the programmatic management of drug-resistant tuberculosis:
} 2011 update

D. Falzon, E. Jaramillo, H.J. Schünemann, M. Arentz, M. Bauer, J. Bayona, L. Blanc, J.A. Caminero, C.L. Daley, C. Duncombe, C. Fitzpatrick, A. Gebhard, H. Getahun, M. Henkens, T.H. Holtz, J. Keravec, S. Keshavjee, A.J. Khan, R. Kulier, V. Leimane, C. Lienhardt, C. Lu, A. Mariandyshev, G.B. Migliori, F. Mirzayev, C.D. Mitnick, P. Nunn, G. Nwagboniwe, O. Oxlade, D. Palmero, P. Pavlinac, M.I. Quelapio, M.C. Raviglione, M.L. Rich, S. Royce, S. Rüsch-Gerdes, A. Salakaia, R. Sarin, D. Sculier, F. Varaine, M. Vitoria, J.L. Walson, F. Wares, K. Weyer, R.A. White and M. Zignol

ABSTRACT: The production of guidelines for the management of drug-resistant tuberculosis (TB) fits the mandate of the World Health Organization (WHO) to support countries in the reinforcement of patient care.

WHO commissioned external reviews to summarise evidence on priority questions regarding casefinding, treatment regimens for multidrug-resistant TB (MDR-TB), monitoring the response to MDR-TB treatment, and models of care. A multidisciplinary expert panel used the Grading of Recommendations Assessment, Development and Evaluation (GRADE) approach to develop recommendations.

The recommendations support the wider use of rapid drug susceptibility testing for isoniazid and rifampicin or rifampicin alone using molecular techniques. Monitoring by sputum culture is important for early detection of failure during treatment. Regimens lasting $\geqslant \mathbf{2 0}$ months and containing pyrazinamide, a fluoroquinolone, a second-line injectable drug, ethionamide (or prothionamide), and either cycloserine or $p$-aminosalicylic acid are recommended. The guidelines promote the early use of antiretroviral agents for TB patients with HIV on second-line drug regimens. Systems that primarily employ ambulatory models of care are recommended over others based mainly on hospitalisation.

Scientific and medical associations should promote the recommendations among practitioners and public health decision makers involved in MDR-TB care. Controlled trials are needed to improve the quality of existing evidence, particularly on the optimal composition and duration of MDR-TB treatment regimens.

KEYWORDS: Ambulatory care facilities, diagnosis, drug therapy, guideline, multidrug-resistant tuberculosis

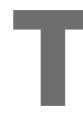

his article reproduces the recommendations of the update of the World Health Organization (WHO) Guidelines for the programmatic management of drug-resistant tuberculosis [1] released in June 2011. The guidelines were developed in compliance with the requirements of the WHO Guidelines Review Committee for evidence gathering, assessment and formulation of recommendations. Some of the text and the tables presented in this article are reproduced from the guidelines [1] and are presented with the permission of WHO.

Tuberculosis (TB) control in the world today must face the challenge posed by the global spread of Mycobacterium tuberculosis strains that are resistant to standard anti-TB drugs $[2,3]$. It is estimated that $\sim 3 \%$ of incident new TB cases in the world have
AFFILIATIONS

For author affiliation details, please refer to the Acknowledgements section.

CORRESPONDENCE

D. Falzon

Stop TB Dept

World Health Organization

20

Av. Appia

CH-1211 Geneva 27

Switzerland

E-mail: falzond@who.int

Received:

May 012011

Accepted after revision:

June 112011 
multidrug-resistant TB (MDR-TB), defined as resistance to at least isoniazid and rifampicin, the two most effective anti-TB drugs [4]. Around 440,000 MDR-TB cases (95\% CI 390,000$510,000)$ are estimated to emerge annually among new and retreated TB patients. The frequency of MDR-TB varies according to region and is much higher among previously treated patients. Amongst the vast majority of MDR-TB patients, very little is known about their access to quality care. Treatment of MDR-TB is complex and uses toxic drugs that must be administered for a longer duration than for drug-susceptible TB patients, with a lower likelihood of treatment success [5].

In 2009, in recognition of the threat posed by drug-resistant TB to global public health security, the World Health Assembly urged Member States to achieve universal access to diagnosis and treatment of patients with this form of disease [6]. The $\mathrm{WHO}$ was mandated to provide technical support to countries for the development and implementation of national frameworks of care for drug-resistant TB patients. The production of guidelines for the programmatic management of drug-resistant TB is part of this role. WHO has previously developed guidelines on this subject, which were based on an assessment of available evidence and best practice by a large group of TB specialists [7, 8]. In 2008, an Emergency Update of the guidelines was published, which expired in 2010. Here, we report on the 2011 update of the guidelines [1], which was developed through a coordinated process that began in 2009. The guidelines target priority areas in drug-resistant TB care. They followed a careful process of systematic retrieval and synthesis of evidence in preparation for the formulation of recommendations by a multidisciplinary expert panel (Guideline Development Group, see Acknowledgements). The panel included TB practitioners, public health professionals, representatives of professional societies, National TB Control Programme staff and guideline methodologists, as well as members of civil society and nongovernmental organisations who provided technical support, and WHO staff. A second group composed of National TB Control Programme staff, WHO regional advisers, clinicians and public health experts was appointed to serve in a peer-review capacity as an External Review Group (see Acknowledgements).

\section{MATERIAL AND METHODS}

\section{Defining the scope of the updated guidelines ("scoping")}

The 2008 Emergency Update [8] of the guidelines identified areas of controversy in which guidance in policy and practice was to be prioritised in future editions of the guidelines. In early 2009, an evaluation of the first two versions of the guidelines was conducted via a user questionnaire [9]. The members of the Guideline Development Group discussed the findings of these two versions and decided to limit the scope of the guidelines to: 1) case-finding (rapid molecular tests for drug resistance, and the investigation of contacts and other high-risk groups); 2) MDR-TB treatment regimens and duration in HIV-positive and HIV-negative patients; 3) monitoring during treatment; and 4) models of care.

This process was translated into the following seven specific questions, which were formulated using PICO (Population, Intervention, Comparator to the intervention, and Outcome) [10] or a similar format.

1) At what prevalence of MDR-TB in any group of TB patients is rapid drug susceptibility testing warranted to detect resistance to rifampicin and isoniazid or rifampicin alone on all patients in the group at the time of TB diagnosis, in order to prescribe appropriate treatment at the outset?

2) Among patients with MDR-TB receiving appropriate treatment in settings with reliable direct microscopy, is monitoring using sputum smear microscopy alone, rather than sputum smear and culture, more or less likely to lead to the relevant outcomes listed in table 1 ?

3) When designing regimens for patients with MDR-TB, is the inclusion of specific drugs (with or without documented susceptibility) more or less likely to lead to the relevant outcomes listed in table 1 ?

4) When designing regimens for patients with MDR-TB, is the inclusion of fewer drugs in the regimen (depending on the drug used, the patient's history of using the drug and isolate susceptibility) more or less likely to lead to the relevant outcomes listed in table 1 ?

5) In patients with MDR-TB, is shorter treatment, compared with the duration currently recommended by $\mathrm{WHO}$, more or less likely to lead to the relevant outcomes listed in table 1 ?

6) In patients with HIV infection and drug-resistant TB who are receiving antiretroviral therapy (ART), is the use of drugs with overlapping and potentially additive toxicities, compared with their avoidance, more or less likely to lead to the relevant outcomes listed in table 1 ?

7) Among patients with MDR-TB, is ambulatory therapy compared with in-patient treatment more or less likely to lead to the relevant outcomes listed in table 1 ?

The External Review Group also provided input into the design and content of the questions. The Guideline Development Group then selected and scored outcomes to determine those which were critical or important for making decisions on recommendations and on which data were to be sought during evidence retrieval and synthesis (table 1).

\section{Reviewing the evidence}

Data sources

Between October 2009 and May 2010, WHO commissioned teams from leading academic centres (see Acknowledgements) to review and compile evidence for each of the questions through a series of systematic reviews of the literature using methods suggested by the Cochrane Collaboration [11]. The teams screened the titles, abstracts and full text of potentially relevant papers using key subject words and text words. The search was not limited by study type or by a time period. In addition, the teams contacted article authors and consulted the Guideline Development Group members to identify studies that were missing or in progress. Individual patient data were collected from authors of published studies to address questions dealing with bacteriology and treatment regimen (questions 2-6). Modelling methods were used for questions 1 and 2. The question on models of care (question 7) was addressed by a review of published and unpublished studies with economic evaluation of MDR-TB patients on treatment.

\section{Analysis}

Where possible, relative effects (hazard ratios, relative risks or odds ratios of an event) were calculated using pooled data 


\section{TABLE 1 What are the most important outcomes to consider when making decisions on testing and treatment strategies for drug-resistant tuberculosis (TB)?}

1) Cure (treatment failure)

2) Prompt initiation of appropriate treatment

3) Avoiding the acquisition or amplification of drug resistance

4) Survival (death)

5) Staying disease-free after treatment; sustaining a cure (relapse)

6) Case holding so the TB patient remains adherent to treatment (default or treatment interruption due to non-adherence)

\section{7) Population coverage or access to appropriate treatment of drug-resistant TB}

8) Smear or culture conversion during treatment

9) Accelerated detection of drug resistance

10) Avoid unnecessary treatment for MDR-TB

11) Population coverage or access to diagnosis of drug-resistant TB

12) Prevention or interruption of transmission of drug-resistant TB to other people, including other patients and healthcare workers

13) Shortest possible duration of treatment

14) Avoiding toxicity and adverse reactions from TB drugs

15) Cost to patient, including direct medical costs as well as others, such as transportation and lost wages due to disability

16) Resolution of TB signs and symptoms; ability to resume usual life activities

17) Interaction of TB drugs with non-TB medications

18) Cost to the TB programme
8.7

8.3

8.1

7.9

7.6

7.6

7.5

7.4

7.4

7.2

7.1

6.9

6.7

6.5

6.4

6.3

5.6

5.4
Critical

Critical

Critical

Critical

Critical

Critical

Critical

Critical

Critical

Critical

Critical

Important but not critical

Important but not critical Important but not critical Important but not critical

Important but not critical Important but not critical Important but not critical

Members of the Guideline Development Group submitted scores for TB outcomes which they considered to be the most critical when making decisions on drug-resistant TB management. Members were asked to take a societal perspective in rating the outcomes. Rating by relative importance was on an incremental scale, as follows. 1-3 points: not important for making recommendations on choice of testing and treatment strategies for drug-resistant TB (none of the outcomes was scored in this category); 4-6 points: important but not critical for making recommendations on choice of testing and treatment strategies; 7-9 points: critical for making recommendations on choice of testing and treatment strategies. MDR-TB: multidrug-resistant TB.

from the studies included. In two of the analyses, outcome was expressed as the cost per disability-adjusted life year (DALY) averted. The DALY is a summary indicator that expresses the burden of mortality and morbidity in a single value, with perfect health valued at 1 and death at 0 (a year with TB disease is valued at 0.729) [12]. To model drug-susceptibility testing (DST), the cost outcomes estimated included total costs for each DST strategy, incremental cost per MDR-TB case prevented, cost per TB-related death avoided and cost per DALY averted. For the analysis of models of care (question 7), any of the following costs were included: cost from the health service provider's perspective, cost from the patient's perspective (including direct medical costs and indirect costs related to transportation) and total societal cost. Whenever possible, the following outcomes were included: proportion of treatment success, default or long-term deaths (including secondary, default and relapse cases) and case reproduction rate (transmission from primary cases).

\section{Developing the recommendations}

Summaries of evidence and GRADE (Grading of Recommendations Assessment, Development and Evaluation) profiles based on the results of the systematic reviews were prepared for each question using a standard approach [13]. These summaries presented the effect of the intervention on each outcome and the quality of the evidence for each effect, categorised into four

\section{TABLE 2 Quality of evidence and definitions}

Quality of evidence Definition

High $(\oplus \oplus \oplus \oplus)$

Moderate $(\oplus \oplus \oplus \mathrm{)})$

Low $(\oplus \oplus \circ)$ )

Very low $(\oplus \circ \bigcirc)$
Further research is very unlikely to change our confidence in the estimate of effect

Further research is likely to have an important impact on our confidence in the effect and may change the estimate

Further research is very likely to have an important impact on our confidence in the estimate of effect and is

likely to change the estimate

Any estimate of effect is very uncertain 
levels (table 2) [14]. The review teams assessed the quality of evidence using the following criteria: study design, limitations in the study (risk of bias), inconsistency, indirectness (whether the evidence directly answers the question being addressed; see [13] for an explanation of the two types of indirectness), imprecision, publication bias, magnitude of effect, dose-effect relationship, and the effect of residual confounding.

On 25-27 October, 2010, the members of the Guideline Development Group met to develop the recommendations at WHO's Headquarters in Geneva, Switzerland. The teams conducting the reviews presented their findings and the GRADE profiles to the Group. The GRADE profiles allowed the Group members to base their judgments on uniformly summarised evidence. In their deliberations, the Group members judged the strength of the recommendations from the perspective of different users (table 3). The higher the quality of evidence, the more likely it was that it led to a strong recommendation. However, a strong recommendation was possible in the presence of very low-quality evidence, as consideration was given to values and preferences that experts attribute to the target population, the balance between desirable and undesirable consequences of an intervention, and resource implications [14]. The Group reached agreement on the recommendations following discussion.

Throughout the guideline revision, the Guideline Development Group considered that the proper management of drug-resistant TB requires a concerted effort from various components of the National TB Control Programme on all activities of care, including case detection, treatment, prevention, surveillance, monitoring and evaluation of programme performance. In the development of the recommendations, the Group attached importance to the following guiding principles: 1) promotion of universal access to care in low-resource settings; 2) prevention of death and transmission of MDR-TB through early diagnosis; 3) avoidance of harm; and 4) provision of care in a setting acceptable to the patient and which optimises the use of resources.

\section{RECOMMENDATIONS}

11 recommendations were made by the Guideline Development Group regarding diagnosis, treatment, monitoring and models of care.

\section{Recommendation 1. Rapid DST of isoniazid and rifampicin or of rifampicin alone is recommended over conventional testing or no testing at the time of diagnosis of TB, subject to available resources (conditional recommendation, $\oplus \bigcirc \bigcirc \bigcirc / v e r y$ low-quality evidence)}

\section{Remarks}

The effect of different DST strategies was simulated using decisionanalysis modelling [15]. This method can only generate very lowquality evidence. Despite limitations, sensitivity analyses showed that the results were fairly consistent under different conditions.

A DST for isoniazid and rifampicin or rifampicin alone that provides a diagnosis within a day or two of testing was considered rapid for this recommendation. Currently, only molecular tests can detect resistance so quickly, of which two technologies, line probe assay and Xpert MTB/RIF, are recommended for use by WHO. (Xpert MTB/RIF refers to the currently available methodology that employs an automated real-time nucleic acid amplification technology for rapid and simultaneous detection of TB and rifampicin resistance.) The basic assumption is that rapid DST will reduce the delay to the start of appropriate second-line therapy, and thus provide benefit to the patient by increasing cure, decreasing mortality, reducing development of additional drug resistance, and reducing the likelihood of failure and relapse.

Rapid DST performed on all patients before the start of treatment was the most cost-effective strategy for averting deaths and preventing the acquisition of additional resistance. Rapid testing for both isoniazid and rifampicin at diagnosis, rather than later during treatment, was the most cost-effective testing strategy available, starting from a MDR-TB prevalence of $>1 \%$ and an isoniazid resistance (other than MDR-TB) of $>2 \%$. Rapid DST for rifampicin alone could also avert many deaths but might not prevent the acquisition of additional resistance in patients resistant to isoniazid alone.

The influence of resistant strains on secondary transmission was not included in the model and therefore, estimations of reductions in mortality and morbidity from early detection and treatment are likely to be conservative. The increased costs of using the diagnostic test may be offset by a reduction in the amount of conventional laboratory capacity needed.

TABLE 3 Implications of the strength of a recommendation for different users

Perspective

For patients

For clinicians

For policy makers
Strong recommendation

Most individuals in this situation would want the recommended course of action and only a small proportion would not. Formal decision aids are not likely to be needed to help individuals make decisions consistent with their values and preferences

Most individuals should receive the intervention. Adherence to this recommendation according to the guidelines could be used as a quality criterion or performance indicator

The recommendation can be adapted as policy in most situations
Conditional recommendation

The majority of individuals in this situation would want the suggested course of action, but many would not

It should be recognised that different choices will be appropriate for individual patients, and that patients must be helped to arrive at a management decision consistent with their values and preferences. Decision aids may be useful in helping individuals to make decisions consistent with their values and preferences Policy making will require substantial debate and the involvement of various stakeholders 
The Group considered costs to the TB programme to be important but not critical. The recommendation is conditional, in part because of the resources required for implementation. Programmes that cannot adhere to the recommendation for all patients may still apply it to groups at higher risk of MDR-TB or unfavourable outcomes, particularly patients treated for TB in the past or with HIV-associated TB, as has been recommended previously [16].

Detection of rifampicin resistance by Xpert MTB/RIF usually suffices to start a patient on a second-line TB regimen [17]. However, the positive predictive value of Xpert MTB/RIF is low in patient groups in which rifampicin resistance is rare. Therefore, to reduce the possible harms of false-positive results for drug resistance, which include wasted resources and avoidable toxicity from the administration of unnecessary second-line medications, results need to be confirmed by phenotypic DST or line probe assay in these patient groups. This is an important consideration given that access to Xpert MTB/RIF is expected to expand substantially in low-resource countries [18].

Recommendation 2. The use of sputum smear microscopy and culture rather than sputum smear microscopy alone is recommended for the monitoring of patients with MDR-TB during treatment (conditional recommendation, $\oplus \bigcirc \bigcirc \bigcirc / v e r y$ low-quality evidence)

Remarks

The evidence used to assess how best to monitor treatment in MDR-TB patients with the use of sputum smear microscopy and culture in settings with reliable direct microscopy was based on data pooled from 10 published observational studies [19-26] included in two recent reviews [5, 27]. Monthly culture monitoring was used as the reference in all of the analyses. Random-effects Cox proportional hazards models were used to estimate the hazard ratio of failure, comparing monthly culture to alternative monitoring strategies.

The use of monthly sputum smear microscopy and culture performed best at identifying failures earlier. Sputum smear microscopy alone resulted in delayed detection of failure; when performed at monthly rather than two-monthly intervals, it increased the detection of failure slightly (not significant). In patients who were smear-negative at the start of treatment, monthly smear monitoring (compared with culture) resulted in a statistically significantly greater risk of delayed detection of failure compared with smear-positive patients. Stratified estimates by HIV serostatus, body mass index, and extent of disease on chest radiograph were not significantly different $(p>0.05)$.

The related end-points of drug resistance, initiation of appropriate treatment and the acquisition of resistance were not measured. There was no information about reversion or reinfection and no data were available to assess the quality of culture and smear testing. Other methods of evaluating response to treatment, such as clinical indicators or chest radiograph, were not evaluated.

Concomitant use of sputum smear microscopy and culture test results helps identify patients whose bacteriology remains positive or reverts back to positive following initial conversion to negative. This is of use to the clinician in identifying patients likely to fail their treatment as well as to institute infection control measures in a timely manner. There was overall certainty in the Group about the risk of missing or delaying the detection of failure if smear microscopy alone was used instead of culture. Additional benefits would be expected from reducing transmission and development of resistance as well as appropriate changes to the treatment regimens, but these were not explicitly addressed by the analysis.

Delayed detection of failure is expected to increase transmission and increase the probability of acquisition of resistance. The 2008 Emergency Update of the guidelines recommended that MDR-TB patients be monitored through monthly sputum smear microscopy and culture examination prior to culture conversion to negative (defined as two consecutive sets of negative results of sputum smear microscopy and culture from samples collected $\geqslant 30$ days apart) and quarterly culture with monthly smear examination after conversion [8]. Even if monthly culture throughout treatment showed the highest benefit of detecting failures, resource implications are important. The cost of sputum smear testing alone is much lower than for culture and ranged between one fourth to one half of the combined cost of culture and smear testing in studies across different settings reviewed for the guidelines [28-34]. It is likely that this difference may be higher where culture diagnosis is not readily available. More laboratory resources (staff, equipment, utilities) are required to perform culture, and fewer culture laboratories exist in the low-resource conditions of most high-burden countries. In settings where the risk of failure is low, selected patients can be prioritised for monthly culture.

The user should be aware of differences in the quality of culture performance. A false-positive result of culture or direct microscopy of sputum smear could lead to unnecessary continuation or modification of a regimen with increased risk of toxicity. A false-negative culture result may change a treatment decision that was based on suggestive clinical findings and a positive sputum smear microscopy result.

A high value was placed on outcomes such as preventing death, decreasing the transmission of MDR-TB that could result from its delayed diagnosis, and avoiding increased use of resources. The recommendation is conditional in part because of the resources required for its implementation. As direct microscopy of sputum smear can identify the most infectious cases within a very short time, it has added value alongside culture for infection control purposes.

Recommendation 3. In the treatment of patients with MDRTB, a fluoroquinolone should be used (strong recommendation, $\oplus \bigcirc \bigcirc \bigcirc /$ very low-quality evidence)

Recommendation 4. In the treatment of patients with MDR$T B$, a later-generation fluoroquinolone rather than an earliergeneration fluoroquinolone should be used (conditional recommendation, $\oplus \bigcirc \bigcirc \bigcirc /$ very low-quality evidence)

Recommendation 5. In the treatment of patients with MDR$T B$, ethionamide (or prothionamide) should be used (strong recommendation, $\oplus \bigcirc \bigcirc \bigcirc /$ very low-quality evidence)

Recommendation 6. In the treatment of patients with MDR$T B$, four second-line anti-TB drugs likely to be effective (including a parenteral agent from among the second-line injectables kanamycin, amikacin or capreomycin), as well 
as pyrazinamide, should be included in the intensive phase (the initial part of a course of treatment during which a parenteral agent is used) (conditional recommendation, $\oplus \bigcirc \bigcirc \bigcirc / v e r y$ low-quality evidence)

Recommendation 7. In the treatment of patients with MDR-TB, regimens should include at least pyrazinamide, a fluoroquinolone, a parenteral agent (kanamycin, amikacin or capreomycin), ethionamide (or prothionamide), and either cycloserine or p-aminosalicylic acid (PAS) if cycloserine cannot be used (conditional recommendation, $\oplus \circ \bigcirc \bigcirc / v e r y$ low-quality evidence)

Remarks

The evidence used to address the questions on which drugs to include and the number of drugs to be used in regimens for MDR-TB patients was based primarily on studies included in three systematic reviews [5, 27, 35]. Studies published before 1970 and those reporting only extensively drug-resistant TB (XDR-TB) were excluded. The reviewers of these questions pooled individual patient data for a meta-analysis from 32 studies with $>9,000$ treatment episodes for which the authors could be contacted and were willing to share their data (study in preparation by the Collaborative Group for Meta-analysis of Individual Patient Data in MDR-TB). Cohorts included had to have a minimum of 25 subjects treated for MDR-TB, with one or more of the treatment outcomes meeting the standard definitions [36]. Patients with XDR-TB $(n=410)$ were excluded from the analysis as their treatment regimens were not considered to be comparable with those of other MDR-TB patients. None of the cohorts was part of a randomised controlled trial and bias was very likely to be substantial (certain drugs may have only been used for sicker patients). The quality of evidence was judged to be low or very low. While the odds ratios in the analysis were adjusted for age, sex, HIV serostatus, past TB treatment, past MDR-TB treatment and extent of disease, residual confounding was certainly to be expected. Other limitations included incomplete ascertainment of relapse, the under-representation of certain geographical regions, and missing data for some of the variables examined. In many of the studies included, drug regimens were adjusted based on DST results. Findings from this analysis may not necessarily be generalisable to all populations in settings with a high or low prevalence of drug resistance or different levels of resources. Nonetheless, the results of this analysis represented the best available evidence to date for the Group to make recommendations on the composition of treatment regimens.

Use of drugs to which the strain was reportedly susceptible showed some added benefit when compared with their use regardless of susceptibility patterns. Choice of drug would thus depend on the DST of the strain isolated from the patient or close contact with MDR-TB, previous use of the drug in the patient, and frequency of use of the drug or documented background drug resistance in the setting. In applying this observation to clinical practice, it is important to underline the uncertainties around the reproducibility and reliability of DST for pyrazinamide (and ethambutol) [37], as well as the secondline anti-TB drugs other than the parenteral agents and the fluoroquinolones [38].

The analysis showed that in the intensive phase, a regimen with at least four drugs likely to be effective, when adjusted for clinical covariates, all other drugs used concomitantly as well as the total number of susceptible drugs used throughout treatment, was associated with a statistically significant peak in cure with a plateau thereafter.

Data from this analysis did not reveal any second-line parenteral agent (kanamycin, amikacin or capreomycin) to be superior in effect to any other. Given its lower cost, kanamycin would be preferable. Amikacin can be used instead of kanamycin. In an analysis comparing patients who were cured or completed treatment with those who failed or relapsed, capreomycin was shown to be effective in the case of resistance to kanamycin. The use of streptomycin in MDR-TB patients is not recommended given the greater likelihood of ototoxicity and the frequent occurrence of resistance to it among MDR-TB patients.

Fluoroquinolones should always be used unless there is a contraindication. They showed a significant association with cure and this effect was more pronounced in later-generation fluoroquinolones (in this analysis, this refers to levofloxacin $\left(\geqslant 750 \mathrm{mg} \cdot \mathrm{day}^{-1}\right)$, moxifloxacin, gatifloxacin and sparfloxacin), and was highest when used against strains known to be susceptible. Estimates of effects of fluoroquinolones were probably conservative given that patients treated with ciprofloxacin were included in the control group. Ciprofloxacin, even if it may have some anti-TB activity, should not be used [39].

Among the oral bacteriostatic drugs, the association with cure was higher with ethionamide than with cycloserine, which was higher than with PAS. Ethionamide or prothionamide should therefore be included in a regimen unless there is a particular contraindication. Ethionamide showed little effect in patients who were treated previously for MDR-TB. PAS performed the worst in the main analysis. Its use would thus be recommended only if an additional drug is needed to have at least four effective secondline drugs in the regimen, and if ethionamide or cycloserine cannot be used or are unlikely to be effective. Studies of the inh $A$ promoter region mutation (not assessed in the review) may, at an additional cost, guide treatment by identifying strains that are resistant to ethionamide [40]. The data did not allow comparison of outcomes between once daily PAS and divided doses, or the formulation of PAS. Decisions on how to administer PAS should thus rely on a balance between its tolerance in the patient and the resources available to observe doses.

Patients who were treated with Group 5 drugs (including clofazimine, linezolid, amoxicillin/clavulanate, thioacetazone, clarithromycin and imipenem; in the analysis for the guidelines, azithromycin, roxithromycin, high-dose isoniazid and thioridazine were also included under Group 5 when used) were observed to have generally worse outcomes, an effect largely attributed to confounding by indication. When the individual effect of amoxicillin/clavulanate, azithromycin, clarithromycin, clofazimine, roxithromycin and thioacetazone was analysed, no significant association with cure could be discerned. No separate analysis was possible for linezolid and high-dose isoniazid given the small number of cases treated with these agents.

Pyrazinamide showed a slightly added benefit in one of the analyses in which adjustment was made for other medication used concomitantly. Ethambutol was associated with a marginal but statistically significant reduction in the likelihood of cure among patients not previously treated for MDR-TB. As 
in the case of Group 5 drugs, this effect was attributed to confounding rather than a detrimental effect of ethambutol.

The main changes from the 2008 Emergency Update [8] of the guidelines are shown in table 4 . The meta-analysis performed for the 2011 update indicated that a minimum of four drugs was associated with a greater likelihood of success. The decision to recommend an additional drug to the regimen during the intensive phase of treatment was based on expert opinion. The intention is to safeguard against the acquisition of additional resistance, particularly in the case of undetected primary resistance to the four drugs considered to be effective given the unreliable nature of DST for drugs other than parenteral agents and fluoroquinolones. If ethambutol and

TABLE 4 Main changes to the recommendations in the 2008 Emergency Update [8] following the 2011 update of the guidelines

\section{8 emergency update}

\section{Monitoring response to MDR-TB treatment}

Regimen composition

\section{Duration of treatment}

Use of ART in drug-resistant TB patients with HIV

Models of care for managing MDR-TB
Monitoring of MDR-TB patients by monthly sputum smear microscopy and culture examination prior to culture conversion to negative and quarterly culture, with monthly smear examination after conversion

Include at least four anti-TB drugs with either certain, or almost certain, effectiveness during the intensive phase of treatment

Consider adding more drugs in patients with extensive disease or uncertain effectiveness

The regimen should include pyrazinamide and/or ethambutol, one fluoroquinolone, one parenteral agent and second-line oral bacteriostatic anti-TB drugs (no preference of oral bacteriostatic second-line anti-TB drug was made)

Ethambutol may be considered effective and included in the regimen if DST shows susceptibility

Treatment with Group 5 drugs is recommended only if additional drugs are needed to bring the total to four

Use of a parenteral agent for a minimum of 6 months and $\geqslant 4$ months after culture conversion

A minimum total length of treatment of 18 months after culture conversion

The timing of the start of ART was in part determined by CD4 cell count

Programmes are encouraged to incorporate community-based care and support into their national plans

\section{1 update}

Monthly sputum smear and culture throughout treatment is recommended, subject to resource implications, given that it has the highest benefit to detect failure

Include at least four second-line anti-TB drugs likely to be effective, as well as pyrazinamide during the intensive phase of treatment

No evidence found to support the use of more than four second-line anti-TB drugs in patients with extensive disease. Increasing the number of second-line drugs in a regimen is permissible if the effectiveness of some of the drugs is uncertain

The regimen should include pyrazinamide, a fluoroquinolone, a parenteral agent, ethionamide (or prothionamide) and cycloserine, or else PAS if cycloserine cannot be used

Ethambutol may be used but is not included among the drugs making up the standard regimen

Group 5 drugs may be used but are not included among the drugs making up the standard regimen

An intensive phase of 8 months' duration is recommended. The duration may be modified depending on bacteriological status and other indicators of progress on treatment

A total treatment duration of $\geqslant 20$ months is recommended in patients without any previous history of MDR-TB treatment. Patients who have had previous treatment for MDR-TB may need longer treatment. The duration may be modified depending on bacteriological status and other indicators of progress on treatment

ART is recommended for all patients with HIV and drug-resistant TB requiring second-line anti-TB drugs, irrespective of CD4 cell count, as early as possible (within the first 8 weeks) following initiation of anti-TB treatment

Patients with MDR-TB should be treated using mainly ambulatory care rather than models of care based principally on hospitalisation

MDR-TB: multidrug-resistant tuberculosis; TB: tuberculosis; PAS: $p$-aminosalicylic acid; DST: drug-susceptibility testing; ART: antiretroviral therapy. 
Group 5 drugs are used to treat MDR-TB patients, they should not be counted among the main drugs making up the MDR-TB regimen, given the inconclusive evidence on their effectiveness. The principle of using additional drugs for extensive disease could not be supported by the data used for the review.

A slight incremental trend in serious adverse events (SAEs) was discerned as the number of drugs in the continuation phase increased from two to five. This association was not observed during the intensive phase. Data were incomplete but SAEs were more often attributed to oral bacteriostatic drugs (14\%) than to the other drugs evaluated (1-6\%). The long-term potential for SAEs, particularly in children and for the latergeneration fluoroquinolones, remains unknown. However, a Cochrane review assessing fluoroquinolones as additional or substitute drugs in regimens for patients with drug-susceptible and drug-resistant strains found that substituting or adding fluoroquinolones to a regimen had no demonstrable effect on the occurrence of SAEs [39].

As patients with XDR-TB were excluded from the analysis, the recommendations do not necessarily apply to this subgroup of patients. Until better evidence is available to optimise regimens for the treatment of these patients, the same principles used to design MDR-TB regimens should be used, based where possible on the DST pattern of strains from the individual patient, particularly for later-generation fluoroquinolones and second-line parenteral agents. All MDR-TB patients should thus be tested for susceptibility to these two classes of drugs.

The aim of the recommendations contained in this section is to increase the likelihood of cure and reduce the risk of failure, relapse and death. A high value was placed on preventing death and transmission of MDR-TB and a lower value on the potential for SAEs resulting from long-term treatment. As a result, the long-term use of fluoroquinolones was considered to outweigh the higher cost and any possible long-term SAEs. The recommendation was therefore strong. While the use of later-generation fluoroquinolones is generally preferred, a separate recommendation on their use was classified as conditional rather than strong because of uncertainty about the risk of SAEs from the long-term use of these agents.

\section{Recommendation 8. In the treatment of patients with MDR- $T B$, an intensive phase of $\geqslant 8$ months' duration is recommended (conditional recommendation, $\oplus \bigcirc \bigcirc \bigcirc / v e r y$ low-quality evidence)}

Recommendation 9. In the treatment of patients with MDR-TB a total treatment duration of $\geqslant 20$ months is recommended in patients without any previous MDR-TB treatment (conditional recommendation, $\oplus \bigcirc \bigcirc \bigcirc /$ very low-quality evidence)

Remarks

The evidence base used to derive these two recommendations was the same as that used for questions 2 to 4 on regimen composition (recommendations 3 to 7). All data were from observational studies and the quality of evidence was classified as very low. Patients with XDR-TB were also excluded from the analysis. Attempts to control for bias and confounding in the review were also unlikely to have adjusted for all important factors. In particular, patients receiving longer therapy may be those who are sicker. These findings may not be generalisable to all populations in settings with a high or low prevalence of drug resistance or with different levels of resources.

The analysis provided evidence for an association between treatment success and the total length of treatment and the length of the intensive phase. The trend in relative risk for cure over successive months of treatment was studied to determine the optimal minimum duration for both total treatment and the intensive phase. The adjusted relative risk for cure peaked at an intensive phase lasting 7.1-8.5 months. For total treatment duration, the peak occurred at 18.6-21.5 months for patients who had no previous MDR-TB treatment. While the peak occurred later in patients who had been treated for MDR-TB (27.6-30.5 months), no clear incremental trend in success was observed in this patient group and the number of observations was far fewer than for those who had no previous MDR-TB treatment. Most patients may be expected to receive this length of treatment but in some it may have to be modified depending on their bacteriological status and other indicators of progress on treatment.

The recommendations have thus changed from those contained in the 2008 Emergency Update [8], which recommended a treatment duration for MDR-TB patients based on the use of a parenteral agent for a minimum of 6 months and $\geqslant 4$ months past culture conversion, and a minimum total length of treatment of 18 months after culture conversion. The new recommended duration of the intensive phase is 2 months longer than the minimum previously recommended. There is, however, no substantial difference in the total length of treatment being recommended, given that conversion typically takes a few months to occur. The data used for this analysis could not inform whether a minimum duration of the intensive phase after conversion was a determinant of outcome.

The risk of SAEs was observed to increase beyond the first 12 months of treatment but was not correlated with the length of the intensive phase beyond the first 2 months. These trends should be interpreted with caution as they may be confounded by the number of drugs used (independently correlated with SAEs) as well as features of the illness process not accounted for in the measure of extent of disease used in the analysis.

A high value was placed on preventing death and transmission of MDR-TB as a result of failed treatment, as well as avoiding harms and minimising use of resources. The Group placed a lower value on reducing the duration of treatment, while acknowledging that many patients may place a higher value on avoiding a long treatment course due to burden and inconvenience. When selecting the duration of treatment, the analysis allowed a choice to be made within a narrow margin of a few consecutive months, thus reducing the likelihood of prolonging treatment unnecessarily. While shorter regimens would confer clear benefits and be preferred, evidence for the effectiveness of a 9-month regimen for MDR-TB patients has as yet been limited to data from one setting (included in the review) [23]. The Guideline Development Group supports further investigation of safety and effectiveness of shorter regimens using the randomised controlled trial design in order to get stronger evidence for their potential use for the treatment of drug-resistant TB.

Recommendation 10. ART is recommended for all patients with HIV and drug-resistant TB requiring second-line 
anti-TB drugs, irrespective of CD4 cell count, as early as possible (within the first 8 weeks) following initiation of anti-TB treatment (strong recommendation, $\oplus \circ \mathrm{O}$ O/very low-quality evidence)

Remarks

Evidence was reviewed from 10 studies [41-50] to assess patient treatment outcomes when ART and second-line anti-TB drugs were used together. None of the data were from randomised controlled trials. Individual patient data were available for 217 drug-resistant TB patients in total, of whom 127 received ART. The quality of evidence in individual observational studies varied from low to very low quality.

The pooled individual patient data showed a lower risk of death and a higher likelihood of cure and resolution of TB signs and symptoms in patients using ART compared with those not using ART (low-quality evidence). There was very low-quality evidence for other outcomes, which were considered critical or important for decision-making (for example, SAEs from second-line drugs for drug-resistant TB, occurrence of conversion of sputum smear or culture, interactions of ART with anti-TB drugs and default from treatment). Available data did not allow assessment of a number of other outcomes of interest, namely avoiding the acquisition of additional drug resistance, preventing TB transmission, sustaining relapse-free cure, establishing the optimal duration of MDR-TB treatment, avoiding unnecessary MDR-TB treatment, and reducing cost and improving population access to appropriate care.

The strong recommendation for use of ART is based in part on indirect evidence from its use in any patient with active TB that shows large beneficial effects and a very high mortality when ART is not employed [51], particularly in very immunocompromised patients (CD4 cell count $<50$ cells $\cdot \mathrm{mm}^{-3}$ ) $[52,53]$. In the absence of other data specific to patients with drug-resistant
TB receiving second-line anti-TB medication, the decision on when to start ART should be no different from the approach to the HIV-positive drug-susceptible TB patient. ART should thus be initiated regardless of CD4 cell count and as soon as anti-TB treatment is tolerated, ideally as early as 2 weeks and no later than 8 weeks after initiation of anti-TB treatment $[51,54]$.

A high value was placed on outcomes such as preventing early death and TB transmission, and a lower value was placed on the resources required to make ART available to all MDR-TB patients with HIV. The capacity to implement this recommendation will require that more providers be trained specifically in the care of HIV and drug-resistant TB and drug-drug interactions. A substantial increase in the availability of and patients' access to treatment and additional support for ensuring adherence is likely to be necessary. The need for increased integration of HIV and TB care for effective patient management, prompt evaluation of adverse events and caseholding throughout treatment will necessitate more resources. For the benefit of the user, a table of adverse events for which both an ART and an anti-TB drug have been implicated, and could conceivably interact, is presented (table 5).

\section{Recommendation 11. Patients with MDR-TB should be treated using mainly ambulatory care rather than models of care based principally on hospitalisation (conditional recommendation, $\oplus \bigcirc \bigcirc \bigcirc /$ very low-quality evidence)} Remarks

Outcomes from models of MDR-TB care based mainly on clinic-based ambulatory treatment were compared with those using mainly hospital-based in-patient treatment. The data used came from published and unpublished cost-effectiveness studies in four countries (Estonia, Peru [24], the Philippines [25] and the Russian Federation (Tomsk Oblast)). The design of these observational studies did not allow direct comparison of

TABLE 5 Potentially overlapping toxicities of antiretroviral and anti-tuberculosis (TB) drugs (including first-line anti-TB drugs)

Potential toxicity

Peripheral neuropathy

Psychiatric symptoms

Hepatitis

Gastro-intestinal intolerance

Nevirapine, ritonavir-boosted protease inhibitors

Zidovudine, protease inhibitors, didanosine

Renal toxicity

Bone marrow toxicity

Lactic acidosis

Stevens-Johnson syndrome

Arrhythmias/QT prolongation

Rash/pruritus
Efavirenz efavirenz, etravirine, maraviroc

Tenofovir, indinavir

Antiretroviral drugs

Stavudine, didanosine

Zidovudine

Stavudine, didanosine, zidovudine

Nevirapine, efavirenz, etravirine

Anti-TB drugs

Cycloserine, isoniazid, ethambutol, fluoroquinolones, streptomycin, kanamycin, amikacin, capreomycin, viomycin ethionamide/prothionamide, linezolid

Cycloserine, isoniazid, fluoroquinolones, ethionamide/ prothionamide,

Pyrazinamide, isoniazid, rifampin/rifabutin, PAS, ethionamide/prothionamide, fluoroquinolones

Ethionomide/prothionomide, PAS, pyrazinamide, isoniazid rifampin, ethambutol, clofazimine

Streptomycin, kanamycin, capreomycin, amikacin, viomycin, rifampin

Linezolid, rifampin/rifabutin Linezolid

Thioacetazone, cycloserine, linezolid, ethambutol, streptomycin

Fluoroquinolones

Rifampin/rifabutin, pyrazinamide

PAS: p-aminosalicylic acid. 
effects between models of care. Given that none of the studies were randomised controlled trials, the evidence was considered to be of very low quality. Cost-effectiveness was modelled for all possible WHO Member States in a probabilistic analysis of the data from the four countries [55].

Cost varied widely across the modelled settings. The cost per DALY averted by an ambulatory model in one setting was sometimes higher than the cost per DALY averted by a hospitalisation model in another setting. However, cost per DALY averted was lower under outpatient-based care than under in-patient-based care in $\geqslant 90 \%$ of the settings for which cost-effectiveness was modelled. The variation in cost-effectiveness among settings correlated most strongly with the variation in the cost of general healthcare services and other non-drug costs. There was no evidence to show that treatment in a hospital-based model of care leads to a more favourable treatment outcome.

The overall cost-effectiveness of care for a patient receiving treatment for MDR-TB can be improved with an ambulatory model. The benefits, when compared with hospitalisation models, include reduced resource use and at least as many deaths avoided among primary and secondary cases. This result is based on clinic-based ambulatory treatment (patients attending a healthcare facility); in some settings, home-based ambulatory treatment (provided by a worker in the community) might improve cost-effectiveness even further. One of the studies of ambulatory care dated from a time when the regimen drug combinations were not yet optimised, so the success achieved was probably inferior to that which can be accomplished with the regimens in use today.

In addition to reducing or avoiding hospitalisation where possible and prioritising community-care approaches for TB management, exposure to people who are infectious can be minimised by reducing the number of outpatient visits and avoiding overcrowding in wards and waiting areas [56]. The benefit of reduced transmission with an ambulatory model can only be achieved if proper infection control measures are in place in both the home and the clinic.

There may be some important barriers to accessing clinic-based ambulatory care, including distance to travel and other costs to individual patients. Shifting costs from the service provider to the patient has to be avoided, and implementation may need to be accompanied by appropriate enablers. While placing patients on adequate therapy would be expected to decrease the bacterial load and transmission of drug-resistant TB, infection control measures for home- and clinic-based measures will need to be part of an ambulatory model of care to decrease the risk of transmission in households, the community and clinics. TB control programmes will have to consider whether they are capable of reallocating resources from hospital to ambulatory care support in order to undertake the necessary changes in patient management. The choice between these options will affect the feasibility of implementing the recommendation in a particular programme.

A high value was placed on conserving resources and on patient outcomes, such as preventing death and transmission of MDR-TB as a result of delayed diagnosis and in-patient treatment. Admission to hospitals for patients may have important social and psychological consequences that need to be taken into account. However, there should always be provision for a back-up facility to manage patients who need in-patient treatment. This may be necessary in certain patient groups at particular risk, such as children during the intensive phase, among whom close monitoring may be required for a period of time.

\section{CONCLUSIONS}

As MDR-TB treatment programmes scale up globally, it becomes critical for treating clinicians to base their practice on the best available evidence. The recommendations for MDR-TB care and control in the new guidelines have been developed following the systematic examination of available evidence on the most salient questions in this area. Although the recommendations on composition and duration of treatment are now based on a meta-analysis of a large set of observations, the quality of all evidence in these studies varied from low to very low. The paucity of costing data has limited the number of studies that could be included to assess the performance of different models of care.

Whilst there have been no drastic changes in the recommendations from the previous guidelines, some changes and the presentation of the evidence on which the recommendations are based will contribute to the dual goals of improving access to care and treatment success. Rapid molecular testing for isoniazid and rifampicin is advisable even in previously untreated patients if resources make it possible. Monthly culture for the monitoring of treatment response is preferred. An intensive phase of 8 months' duration is conditionally recommended instead of the previous minimum of 6 months. The addition of pyrazinamide to a minimum of four second-line anti-TB drugs that are likely to be effective is recommended. The use of fluoroquinolones and ethionamide is strongly recommended. Later-generation fluoroquinolones are preferred. The contribution of ethambutol and Group 5 drugs in MDR-TB treatment remains unclear. All patients with drug-resistant TB and HIV who are on second-line anti-TB medications should be placed on ART as soon as they can tolerate it. Systems that primarily employ ambulatory models of care are recommended over others based mainly on hospitalisation.

The process of developing these guidelines revealed some important gaps in the knowledge that should be addressed in future research, particularly in the context of large-scale expansion of treatment for patients with drug-resistant TB. These include a lack of high- or moderate-quality evidence from randomised controlled trials for the optimisation of treatment regimen in patients with drug-resistant TB, particularly for determining the best combination of drugs and treatment duration. In addition, evidence was lacking on: 1) the treatment of paediatric MDR-TB; 2) the best drug regimens for treatment of patients with isoniazid resistance, XDR-TB or non-MDR-TB poly-drug resistance; 3) effective chemoprophylaxis for contacts of MDR-TB cases; and 4) therapy for symptomatic relief from adverse reactions linked with second-line anti-TB drugs.

In anticipation of the availability of new anti-TB drugs in the near future, and the development of novel diagnostic tools, these recommendations require a strong commitment by national $\mathrm{TB}$ 
programmes to ensure their implementation at all levels. WHO, in collaboration with its technical and implementing partners, will strive to communicate them through different means. As in the past, the support of the European Respiratory Society (ERS) [57] and other leading scientific groups in respiratory medicine, including the American Thoracic Society (ATS), the Pan African Thoracic Society (PATS), the International Union Against Tuberculosis and Lung Disease (The UNION), the American College of Chest Physicians (ACCP), the Asian Pacific Society of Respirology (APSR) and ALAT (Asociación Latinoamericana del Tórax), will be crucial to the effective spread of the key messages and to assist countries to adapt the recommendations and evaluate their implementation.

\section{SUPPORT STATEMENT}

Funding for the meetings and reviews involved in the updating of the guidelines came entirely from the United States Agency For International Development (USAID).

\section{STATEMENT OF INTEREST}

Statements of interest for J. Bayona, C.L. Daley, C.D. Mitnick and M.I. Quelapio can be found at www.erj.ersjournals.com/site/misc/ statements.xhtml

\section{ACKNOWLEDGEMENTS}

The affiliation details of the authors and Guideline Development Group (presented in italics) are as follows. D. Falzon: Stop TB Dept, WHO, Geneva, Switzerland. E. Jaramillo: Stop TB Dept, WHO, Geneva, Switzerland. H.J. Schünemann: McMaster University Health Sciences, Hamilton, ON, Canada. M. Arentz: University of Washington, Seattle, WA, USA. M. Bauer: University of McGill, Montreal, QC, Canada. J. Bayona: Socios En Salud Sucursal, Lima, Peru. L. Blanc: Stop TB Dept, WHO, Geneva, Switzerland. J.A. Caminero: Pneumology Dept, University General Hospital of Gran Canaria, Las Palmas, Spain, and The UNION, Paris, France. C.L. Daley: National Jewish Health, Denver, CO, USA. C. Duncombe: HIV Dept, WHO, Geneva, Switzerland. C. Fitzpatrick: Stop TB Dept, WHO, Geneva, Switzerland. A. Gebhard: KNCV Tuberculosis Foundation, The Hague, The Netherlands. H. Getahun: Stop TB Dept, WHO, Geneva, Switzerland. M. Henkens: Médecins Sans Frontières, Paris, France. T.H. Holtz: CDC, Bangkok, Thailand. J. Keravec: Management Sciences for Health, Arlington, VA, USA. S. Keshavjee: Partners In Health, Boston, MA, USA. A.J. Khan: Indus Hospital, Karachi, Pakistan. R. Kulier: Research Policy and Cooperation, WHO, Geneva, Switzerland. V. Leimane: State Infectology Center, Clinic of Tuberculosis and Lung Diseases, Riga, Latvia. C. Lienhardt: Stop TB Partnership, WHO, Geneva, Switzerland. C. Lu: Harvard Medical School, Boston, MA, USA. A. Mariandyshev: Northern State Medical University, Arkhangelsk, Russian Federation. G.B. Migliori: WHO Collaborating Centre for TB and Lung Diseases, Fondazione S. Maugeri, Tradate, Italy. F. Mirzayev: Stop TB Dept, WHO, Geneva, Switzerland. C.D. Mitnick: Harvard Medical School, Boston, MA, USA. P. Nunn: Stop TB Dept, WHO, Geneva, Switzerland. G. Nwagboniwe: Alliance for Hope, Asaba, Nigeria. O. Oxlade: University of McGill, Montreal, QC, Canada. D. Palmero: Hospital Muniz, Buenos Aires, Argentina. P. Pavlinac: University of Washington, Seattle, WA, USA. M.I. Quelapio: Tropical Disease Foundation, Manila, Philippines. M.C. Raviglione: Stop TB Dept, WHO, Geneva, Switzerland. M.L. Rich: Partners In Health, Boston, MA, USA. S. Royce: PATH (Programme for Appropriate Technology in Health), Seattle, WA, USA. S. Rüsch-Gerdes: National Reference Center for Mycobacteria, Borstel, Germany. A. Salakaia: Management Sciences for Health, Arlington, VA, USA. R. Sarin: LRS Institute of TB and Allied Diseases, New Delhi, India. D. Sculier: Stop TB Dept, WHO, Geneva, Switzerland. F. Varaine: Médecins Sans Frontières, Paris, France. M. Vitoria: HIV Dept, WHO, Geneva, Switzerland. J.L. Walson:
University of Washington, Seattle, WA, USA. F. Wares: Stop TB Dept, WHO, Geneva, Switzerland. K. Weyer: Stop TB Dept, WHO, Geneva, Switzerland. R.A. White: Harvard School of Public Health, Boston, MA, USA. M. Zignol: Stop TB Dept, WHO, Geneva, Switzerland.

The lead authors of this article were D. Falzon, E. Jaramillo and H.J Schünemann. G.B. Migliori contributed to the adaptation of the text from the guidelines and the initial review of the manuscript. All other authors contributed to different degrees in the ideation, development of the questions and outcomes, drafting of the recommendations and by commenting on an advanced draft of this article. The authors are grateful to other workers who contributed data for the reviews as cited in the references, and in particular the Collaborative Group for MetaAnalysis of Individual Patient Data in MDR-TB, led by R. Menzies (University of McGill, Montreal, QC, Canada) [36].

E. Skachkova (Federal Center of TB Monitoring, Moscow, Russian Federation) was a member of the Guideline Development Group.

The academic centres reviewing the evidence for these guidelines were: University of McGill, Montreal, QC, Canada (M. Bauer, R. Menzies, O. Oxlade); Harvard Medical School (C. Lu, C.D. Mitnick, and Harvard School of Public Health (R.A. White), Boston, MA, USA; University of California San Francisco, San Francisco, CA, USA (G. Kennedy, G. Rutherford, K. Steingart); University of Washington, Seattle, WA, USA (M. Arentz, D. Horne, P. Pavlinac, J.L. Walson).

The following members of the External Review Group also contributed to the production of the guidelines: S. Baghdadi, M. Becerra, V. Bhatia, M. Dara, M. del Granado, R. Granich, L. Mvusi, N. Nair, N. Ndjeka, W. Nkhoma, K. Osuga, H.S. Schaaf, C. van Weezenbeek, I. Vasilyeva, W. Xie Xiu, and R. Zaleskis. At WHO Stop TB Dept, M. Grzemska and C. Gunneberg advised the initial stages of the development of the guidelines, K. Ciceri provided editorial support, and C. Chevalley assisted the coordination process of the revision.

\section{REFERENCES}

1 World Health Organization. Guidelines for the programmatic management of drug-resistant tuberculosis, 2011 update. Geneva, WHO, 2011. Available from: http:/ / whqlibdoc.who.int/publications/ 2011/9789241501583_eng.pdf

2 Towards universal access to diagnosis and treatment of multidrug-resistant and extensively drug-resistant tuberculosis by 2015. WHO progress report 2011. Geneva, WHO, 2011 (WHO/ HTM/TB/2011.3).

3 Multidrug and extensively drug-resistant TB (M/XDR-TB): 2010 global report on surveillance and response. Geneva, WHO, 2010 (WHO/HTM/TB/2010.3).

4 Global tuberculosis control: WHO report 2010. Geneva, WHO, 2010 (WHO/HTM/TB/2010.7).

5 Orenstein EW, Basu S, Shah NS, et al. Treatment outcomes among patients with multidrug-resistant tuberculosis: systematic review and meta-analysis. Lancet Infect Dis 2009; 9: 153-161.

6 Resolution WHA62.15. Prevention and control of multidrugresistant tuberculosis and extensively drug-resistant tuberculosis. In: Sixty-second World Health Assembly: Resolutions and Decisions, Annexes. Geneva, WHO, 2009 (WHA62/2009/REC/ 1). Available from: apps.who.int/gb/ebwha/pdf_files/WHA62REC1/WHA62_REC1-en.pdf

7 Guidelines for the programmatic management of drug-resistant tuberculosis. 1st Edn. Geneva, WHO, 2006 (WHO/HTM/TB/ 2006.361).

8 Guidelines for the programmatic management of drug-resistant tuberculosis, Emergency update 2008. Geneva, WHO, 2008 (WHO/HTM/TB/2008.402).

9 Shukhobodskaya E, Falzon D, Jaramillo E. Evaluation of the WHO guidelines on programmatic management of drug-resistant 
tuberculosis. Available from: www.worldlunghealth.org/Conf2009/ website/assets/files/Abstract_Book_2009_Web.pdf

10 Richardson WS, Wilson MC, Nishikawa J, et al. The well-built clinical question: a key to evidence-based decisions. ACP J Club 1995; 123: A12-A13.

11 Higgins JPT, Green S. Cochrane Handbook for Systematic Reviews of Interventions. Chichester, John Wiley \& Sons, 2008.

12 Global burden of disease 2004 update: disability weights for diseases and conditions. Geneva, WHO, 2004. Available from: www.who. int/healthinfo/global_burden_disease/GBD2004_DisabilityWeights. pdf

13 Guyatt GH, Oxman AD, Kunz R, et al. What is "quality of evidence" and why is it important to clinicians? BMJ, 20083; 336: 995-998.

14 Guyatt GH, Oxman AD, Kunz R, et al. Going from evidence to recommendations. BMJ 2008; 336: 1049-1051.

15 Oxlade O, Falzon D, Menzies D. The impact and cost-effectiveness of strategies to detect drug-resistant tuberculosis. Eur Respir J 2011; (In press).

16 Guidelines for treatment of tuberculosis. 4th Edn. Geneva, WHO, 2009 (WHO/HTM/TB/2009.420).

17 Rapid Implementation of the Xpert MTB/RIF diagnostic test. Technical and operational "how-to" practical considerations. Geneva, WHO, 2011. Available from: whqlibdoc.who.int/publications/2011/ 9789241501569_eng.pdf

18 Boehme CC, Nabeta P, Hillemann D, et al. Rapid molecular detection of tuberculosis and rifampin resistance. $N$ Engl J Med 2010; 363: 1005-1015.

19 Migliori GB, Lange C, Centis R, et al. Resistance to second-line injectables and treatment outcomes in multidrug-resistant and extensively drug-resistant tuberculosis cases. Eur Respir J 2008; 31: $1155-1159$.

20 Cox H, Kebede Y, Allamuratova S, et al. Tuberculosis recurrence and mortality after successful treatment: impact of drug resistance. PLoS Med 2006; 3: e384.

21 Holtz TH, Lancaster J, Laserson KF, et al. Risk factors associated with default from multidrug-resistant tuberculosis treatment, South Africa, 1999-2001. Int J Tuberc Lung Dis 2006; 10: 649-655.

22 CDC, Partners In Health/NTP Peru, Partners In Health/Tomsk Prison \& Civilian TB Services, NTP Latvia, NTP Estonia, TDF/ NTP Philippines, WHO. Case-based data collection: First 5 DOTSPlus Projects, 2000-2004 [dataset].

23 Van Deun A, Maug AK, Salim MA, et al. Short, highly effective, and inexpensive standardized treatment of multidrugresistant tuberculosis. Am J Respir Crit Care Med 2010; 182: 684-692.

24 Suárez PG, Floyd K, Portocarrero J, et al. Feasibility and costeffectiveness of standardised second-line drug treatment for chronic tuberculosis patients: a national cohort study in Peru. Lancet 2002; 359: 1980-1989.

25 Tupasi TE, Gupta R, Quelapio MI, et al. Feasibility and costeffectiveness of treating multidrug-resistant tuberculosis: a cohort study in the Philippines. PLoS Med 2006; 3: e352.

26 The feasibility and efficiency of controlling MDR-TB using the DOTS-Plus strategy in the Russian Federation. Geneva, WHO, 2005 (WHO/HTM/TB/2005.357C).

27 Johnston JC, Shahidi NC, Sadatsafavi M, et al. Treatment outcomes of multidrug-resistant tuberculosis: a systematic review and metaanalysis. PLoS One 2009; 4: e6914.

28 Dowdy DW, O'Brien MA, Bishai D. Cost-effectiveness of novel diagnostic tools for the diagnosis of tuberculosis. Int J Tuberc Lung Dis 2008; 12: 1021-1029.

29 Dowdy DW, Lourenço MC, Cavalcante SC, et al. Impact and costeffectiveness of culture for diagnosis of tuberculosis in HIVinfected Brazilian adults. PLoS One 2008; 3: e4057.

30 Menzies D, Oxlade O, Lewis M. Costs for tuberculosis care in Canada. Ottawa, Public Health Agency of Canada, 2006.
31 The efficiency of TB laboratory services in the Russian Federation. Policy Brief Number 5. Geneva, WHO, 2005 (WHO/HTM/TB/ 2005.357E).

32 Albert $\mathrm{H}$. Economic analysis of the diagnosis of smear-negative pulmonary tuberculosis in South Africa: incorporation of a new rapid test, FASTPlaqueTB, into the diagnostic algorithm. Int $J$ Tuberc Lung Dis 2004; 8: 240-247.

33 Kamolratanakul P, Hiransithikul N, Singhadong N. Cost analysis of different types of tuberculosis patients at tuberculosis centers in Thailand. Southeast Asian J Trop Med Public Health 2002; 33: 321-330.

34 The Economics of TB Drug Development. The Global Alliance for TB Drug Development 2001. Available from: www.tballiance.org/ downloads/publications/TBA_Economics_Report.pdf

35 Akçakır Y. Correlates of treatment outcomes of multidrugresistant tuberculosis (MDR-TB): a systematic review and metaanalysis. MSc Thesis. Montreal, McGill University, 2010.

36 Laserson KF, Thorpe LE, Leimane V, et al. Speaking the same language: treatment outcome definitions for multidrug-resistant tuberculosis. Int J Tuberc Lung Dis 2005; 9: 640-645.

37 Framework for implementing new tuberculosis diagnostics. Geneva, WHO, 2010. Available from: www.who.int/tb/laboratory/ whopolicyframework_july10_revnov10.pdf

38 Policy guidance on drug susceptibility testing (DST) of second-line anti-tuberculosis drugs. Geneva, WHO, 2008 (WHO/HTM/TB/ 2008.392). Available from: whqlibdoc.who.int/hq/2008/WHO_ HTM_TB_2008.392_eng.pdf

39 Ziganshina LE, Squire SB. Fluoroquinolones for treating tuberculosis. Cochrane Database Syst Rev 2008; 1: CD004795.

40 Lee H, Cho SN, Bang HE, et al. Exclusive mutations related to isoniazid and ethionamide resistance among Mycobacterium tuberculosis isolates from Korea. Int J Tuberc Lung Dis 2000; 4: 441-447.

41 Burgos M, Gonzalez LC, Paz EA, et al. Treatment of multidrugresistant tuberculosis in San Francisco: an outpatient-based approach. Clin Infect Dis 2005; 40: 968-975.

42 Dheda K, Shean K, Zumla A, et al. Early treatment outcomes and HIV status of patients with extensively drug-resistant tuberculosis in South Africa: a retrospective cohort study. Lancet 2010; 375: 1798-1807.

43 Eker B, Ortmann J, Migliori GB, et al. Multidrug- and extensively drug-resistant tuberculosis, Germany. Emerg Infect Dis 2008; 14: 1700-1706.

44 El Sahly HM, Teeter LD, Pawlak RR, et al. Drug-resistant tuberculosis: a disease of target populations in Houston, Texas. J Infect 2006; 53: 5-11.

45 Leimane V, Dravniece G, Riekstina V, et al. Treatment outcome of multidrug/extensively drug-resistant tuberculosis in Latvia, 20002004. Eur Respir J 2010; 36: 584-593.

46 Migliori GB, Besozzi G, Girardi E, et al. Clinical and operational value of the extensively drug-resistant tuberculosis definition. Eur Respir J 2007; 30: 623-626.

47 Palmero D, Ritacco V, Ambroggi M, et al. [Multidrug-resistant tuberculosis in AIDS patients at the beginning of the millennium.] Medicina (B Aires) 2006; 66: 399-404.

48 Shean KP, Willcox PA, Siwendu SN, et al. Treatment outcome and follow-up of multidrug-resistant tuberculosis patients, West Coast/Winelands, South Africa, 1992-2002. Int J Tuberc Lung Dis 2008; 12: 1182-1189.

49 Varma JK, Nateniyom S, Akksilp S, et al. HIV care and treatment factors associated with improved survival during TB treatment in Thailand: an observational study. BMC Infect Dis 2009; 9: 42.

50 Jamal LF, Guibu IA, Tancredi MV, et al. Reliability and usefulness of TB/HIV co-infection data proceeding from developing countries. International Conference on AIDS 2004. Available 
from: gateway.nlm.nih.gov/MeetingAbstracts /ma?f=102280737. html

51 Antiretroviral therapy for HIV infection in adults and adolescents: recommendations for a public health approach. 2010 revision. Geneva, WHO, 2010.

52 Abdool Karim S, Naidoo K, Padayatchi N, et al. Optimal Timing of ART during TB Therapy: Findings of the SAPiT Trial. 18th Conference on Retroviruses and Opportunistic Infections, Boston, USA, 2011. Available from: www.retroconference.org/2011/ Abstracts/42488.htm

53 Havlir D, Ive $\mathrm{P}$, Kendall M, et al. International randomized trial of immediate $v$ s. early ART in HIV+ patients treated for TB: ACTG 5221 STRIDE study. 18th Conference on Retroviruses and Opportunistic Infections, Boston, USA, 2011. Available from: www.retroconference.org/2011/Abstracts/41152.htm
54 Blanc FX, Sok T, Laureillard D, et al. Significant enhancement in survival with early (2 weeks) vs. late ( 8 weeks) initiation of highly active antiretroviral treatment (HAART) in severely immunosuppressed HIV-infected adults with newly diagnosed tuberculosis. 18th Intl AIDS Conf, Abstract THLBB106, Vienna, Austria, 2010. Available from: www.natap.org/2010/IAS/IAS_91.htm

55 Fitzpatrick C, Floyd K. A systematic review of the cost and costeffectiveness of treatment for multidrug-resistant tuberculosis. Pharmacoeconomics 2011; (In press).

56 WHO Policy on TB infection control in health-care facilities, congregate settings and households. Geneva, WHO, 2009 (WHO/ $\mathrm{HTM} / \mathrm{TB} / 2009.419)$

57 Migliori GB, Loddenkemper R, Blasi F, et al. 125 years after Robert Koch's discovery of the tubercle bacillus: the new XDR-TB threat. Is "science" enough to tackle the epidemic?Eur Respir J 2007; 29: 423-427. 